\title{
North-south anisotropy of galactic cosmic rays observed with the Global Muon Detector Network (GMDN)
}

\author{
K. Munakata ${ }^{a *}$, M. Kozai $^{a}$, C. Kato ${ }^{a}$, T. Kuwabara ${ }^{b}$, M. Rockenbach ${ }^{c}$, A. Dal Lago ${ }^{d}$, N. \\ J. Schuch ${ }^{c}$, H. K. Al Jassare, M. M. Sharma ${ }^{e}$, M. L. Duldig ${ }^{f}$, J. E. Humble ${ }^{f}$, J. W. \\ Bieber $^{g}$, P. Evenson ${ }^{g}$, I. Sabbah ${ }^{h}$ and M. Tokumaru ${ }^{i}$ \\ ${ }^{a}$ Physics Department, Shinshu University, Matsumoto, Nagano 390-8621, Japan. \\ ${ }^{b}$ Graduate School of Science, Chiba University, Chiba City, Chiba 263-8522, Japan. \\ ${ }^{c}$ Southern Regional Space Research Center (CRS/INPE), P.O. Box 5021, 97110-970, Santa Maria, RS, Brazil. \\ ${ }^{d}$ National Institute for Space Research (INPE), 12227-010 São José dos Campos, SP, Brazil. \\ ${ }^{e}$ Physics Department, Kuwait University, P.O. Box 5969 Safat, Kuwait 13060. \\ ${ }^{f}$ School of Physical Sciences, University of Tasmania, Hobart, Tasmania 7001, Australia. \\ ${ }^{g}$ Bartol Research Institute and Department of Physics and Astronomy, University of Delaware, Newark, DE 19716, USA. \\ ${ }^{h}$ Department of Natural Sciences, College of Health Sciences, Public Authority of Applied Education and Training, \\ Kuwait City 72853, Kuwait. \\ ${ }^{i}$ Solar Terrestrial Environment Laboratory, Nagoya University, Nagoya, Aichi 464-8601, Japan. \\ E-mail: kmuna00@shinshu-u.ac.jp, 13st303f@shinshu-u.ac.jp, ckato@shinshu-u.ac.jp, \\ takao@bartol.udel.edu, marlosrs@gmail.com, filhodesun@yahoo.com.br, \\ njschuch@lacesm.ufsm.br, hala.aljassar@ku.edu.kw, madan.sharma@ku.edu.kw \\ Marc.Duldig@utas.edu.au, john. humble@utas.edu.au, jwbieber@bartol. udel. edu, \\ evenson@UDel.Edu, sabbahsom@yahoo.com, tokumaru@stelab.nagoya-u.ac.jp,
}

We analyze the north-south anisotropy (NSA) of galactic cosmic rays observed with the GMDN on an hourly basis and compare with the anisotropy derived from the GG-component of a large multidirectional muon detector at Nagoya, Japan. The NSA is a component of the three dimensional anisotropy vector parallel to the Earth's rotation axis. We find a significant seasonal variation of the NSA from the GG-component indicating the influence of the anisotropy component in the ecliptic plane to the NSA. We calculate the average and standard deviation of daily mean NSAs in the "toward" (T) and "away" (A) IMF sectors separately in each Carrington Rotation between December 1993 and November 2014. It is confirmed that the temporal variations of the NSA observed with the GMDN and GG-component are consistent with each other, as reported earlier. We find the T-A separation between average NSAs in T and A sectors shows a long-term variation with minima (maxima) around the solar activity minima (maxima). The standard deviation in each rotation also shows a similar long-term variation, keeping the ratio of T-A separation to the standard deviation roughly constant thorough out an entire period of analysis. We discuss this in relation with the "success rate" which is introduced as a parameter indicating to what extent we can infer the IMF sector polarity from the sign of the observed NSA.

The 34th International Cosmic Ray Conference,

30 July- 6 August, 2015

The Hague, The Netherlands

${ }^{*}$ Speaker. 


\section{Introduction}

A solar disturbance propagating away from the Sun affects the pre-existing population of galactic cosmic rays (GCRs) in a number of ways. By measuring the anisotropy (or the streaming) of high energy GCR intensity and by using Parker's transport equation of GCRs in the heliosphere [1], we can infer the large-scale spatial gradient of GCR density (or isotropic component of GCR intensity) which reflects the magnetic structure such as interplanetary shocks and magnetic flux ropes in the Interplanetary Coronal Mass Ejection (ICME). Only a global network of detectors can measure the dynamic variation of the first order anisotropy accurately and separately from the temporal variation of the GCR density.

For accurate measurement of the anisotropy, the Global Muon Detector Network (GMDN) was established in March 2006, when a hodoscope type cosmic ray detector using proportional counter tubes was installed in Kuwait and added to the previous network composed of MDs using plastic scintillators at Nagoya in Japan, Hobart in Australia and São Martinho da Serra in Brazil. The detection areas of MDs, except one at Nagoya which has an area of $6 \mathrm{~m} \times 6 \mathrm{~m}$, have been enlarged in several steps and are currently $4 \mathrm{~m}$ x $4 \mathrm{~m}$ at Hobart, $4 \mathrm{~m} \mathrm{x} 8 \mathrm{~m}$ at São Martinho da Serra and $5 \mathrm{~m} \times 5 \mathrm{~m}$ at Kuwait, respectively. The GMDN has been continuously in operation, precisely observing the anisotropy on hourly basis $[2,3]$. The GMDN data are available on the internet for open access at a web page: http://cosray.shinshu-u.ac.jp/crest/DB/Public/Archives/GMDN.php.

The GCR anisotropy vector in three dimensions consists of two components, one lying in the equatorial plane and the other parallel to the Earth's rotation axis. The ecliptic component has been derived from the amplitude and phase of the solar diurnal anisotropy in many papers $[4,5,6]$, while the north-south anisotropy (NSA) parallel to the Earth's rotation axis has been derived from NM and MD data in a couple of different ways [7, 8, 9]. Mori and Nagashima (1979) [10] proposed a way to derive the NSA from the "GG-component" of a single multi-directional MD at Nagoya in Japan. The GG-component is a difference combination between intensities recorded in the geographically north- and south-viewing directional channels and designed to measure the NSA free from the atmospheric temperature effect in the muon count rate [16]. The GG-component has been recognized as a good measure of the NSA. Based on observations of the NSA which varies depending on the orientation of the Interplanetary Magnetic Field (IMF) around the Earth, it has been demonstrated that the GG-component can be used for deriving reliable sector polarity of the IMF which is defined as away (toward) when the IMF directs away from (toward) the Sun [11]. It is also reported that the NSA deduced from the GG-component is consistent with the anisotropy observed with the GMDN $[2,12]$.

It is noted, however, that the GG-component also has a response to the diurnal anisotropy in the equatorial plane. The influence of the diurnal anisotropy to the GG-component can be eliminated by taking an average over one day when the anisotropy is stationary at least over one day, but this elimination may not work if the anisotropy changes dynamically within a day, as often observed with the GMDN $[13,14,3]$. It is important, therefore, to examine whether the NSA derived from the GG-component is consistent with the anisotropy observed by the GMDN which is capable of accurately measuring the anisotropy with better time resolution. In this paper, we analyze average features of the NSA observed with the GMDN and GG-component over 22 years between 1993 and 2014. 


\section{Data analysis and results}

\subsection{Derivation of the three dimensional anisotropy from the GMDN data}

We analyze the pressure corrected hourly count rate $I_{i, j}(t)$ of muons recorded in the $j$-th directional channel of the $i$-th detector in the GMDN at the universal time $t$ and derive three components $\left(\xi_{x}^{\mathrm{GEO}}(t), \xi_{y}^{\mathrm{GEO}}(t), \xi_{z}^{\mathrm{GEO}}(t)\right)$ of the first order anisotropy in the geographic (GEO) coordinate system by best-fitting the following model function to $I_{i, j}(t)$.

$$
\begin{aligned}
I_{i, j}^{f i t}(t)=I_{i, j}^{0}(t) & +\xi_{x}^{\mathrm{GEO}}(t)\left(c_{1 i, j}^{1} \cos \omega t_{i}-s_{1 i, j}^{1} \sin \omega t_{i}\right) \\
& +\xi_{y}^{\mathrm{GEO}}(t)\left(s_{1 i, j}^{1} \cos \omega t_{i}+c_{1 i, j}^{1} \sin \omega t_{i}\right) \\
& +\xi_{z}^{\mathrm{GEO}}(t) c_{1 i, j}^{0}
\end{aligned}
$$

where $I_{i, j}^{0}(t)$ is a parameter representing the contributions from the omnidirectional intensity and the atmospheric temperature effect, $t_{i}$ is the local time at the $i$-th detector, $c_{1 i, j}^{1}, s_{1 i, j}^{1}$ and $c_{1 i, j}^{0}$ are the coupling coefficients and $\omega=\pi / 12$. The coupling coefficients are calculated by using the response function of atmospheric muons to the primary cosmic rays $([15,16,17])$ by assuming a rigidity independent anisotropy. $\xi_{x}^{\mathrm{GEO}}(t)$ and $\xi_{y}^{\mathrm{GEO}}(t)$ are two components of the diurnal anisotropy in the equatorial plane, while $\xi_{z}^{\mathrm{GEO}}(t)$ is the NSA parallel to the Earth's rotation axis. Note that the anisotropy vector in this paper points toward a direction from which the highest GCR flux is measured; i.e., the anisotropy vector is oppositely directed to the GCR streaming vector. In our papers published earlier, the anisotropy vector in the GEO coordinate system derived from the GMDN in equation (2.1) is transformed to the geocentric solar ecliptic (GSE) coordinate system, in which the $z$-component corresponds to the component normal to the ecliptic plane, and corrected for the solar wind convection anisotropy using the solar wind velocity data and for the Compton-Getting anisotropy arising from the Earth's $30 \mathrm{~km} / \mathrm{s}$ orbital motion around the Sun. We then obtain the ecliptic plane component of the anisotropy consisting of components parallel $\left(\xi_{\|}\right)$and perpendicu$\operatorname{lar}\left(\xi_{\perp}\right)$ to the IMF as obtained from the omnitape data and the $z$-component normal.

In the present paper, we calculate average $\xi_{x}^{\mathrm{GEO}}, \xi_{y}^{\mathrm{GEO}}$ and $\xi_{z}^{\mathrm{GEO}}$ in each Carrington Rotation $(\mathrm{CR})$ and subtract them from the hourly values. By using the hourly $\mathrm{x}, \mathrm{y}$ components $\left(B_{x}, B_{y}\right)$ of the IMF vector in the GSE coordinate system recorded in the "omnitape" data [18], we identify the IMF sector as toward (T), if $B_{x}>0$ and $B_{y}<0$ and away (A) if $B_{x}<0$ and $B_{y}>0$. We obtain the daily mean anisotropy vector in T or A sectors in every day by averaging hourly anisotropy vectors in the corresponding sector, if there is more than 5 hour data in $\mathrm{T}$ or $\mathrm{A}$ sector in that day.

\subsection{Derivation of NSA from the GG-component}

The GG-component is a difference combination between intensities recorded in the north- and south-viewing channels of a multi-directional muon detector at Nagoya in Japan and is designed to detect the NSA free from the atmospheric temperature effect $[16,10]$. The GG-component is defined as a sum of two differences, as

$$
G G(t)=\left\{r_{\mathrm{N} 2}(t)-r_{\mathrm{S} 2}(t)\right\}+\left\{r_{\mathrm{N} 2}(t)-r_{\mathrm{E} 2}(t)\right\}
$$

where $r_{X X}(t)$ is the percent deviation of the pressure-corrected muon rate $I_{X X}(t)$ in the directional channel $X X(=N 2, S 2, E 2)$ from the average rate. Three directional channels $(\mathrm{N} 2, \mathrm{~S} 2, \mathrm{E} 2)$ have a 


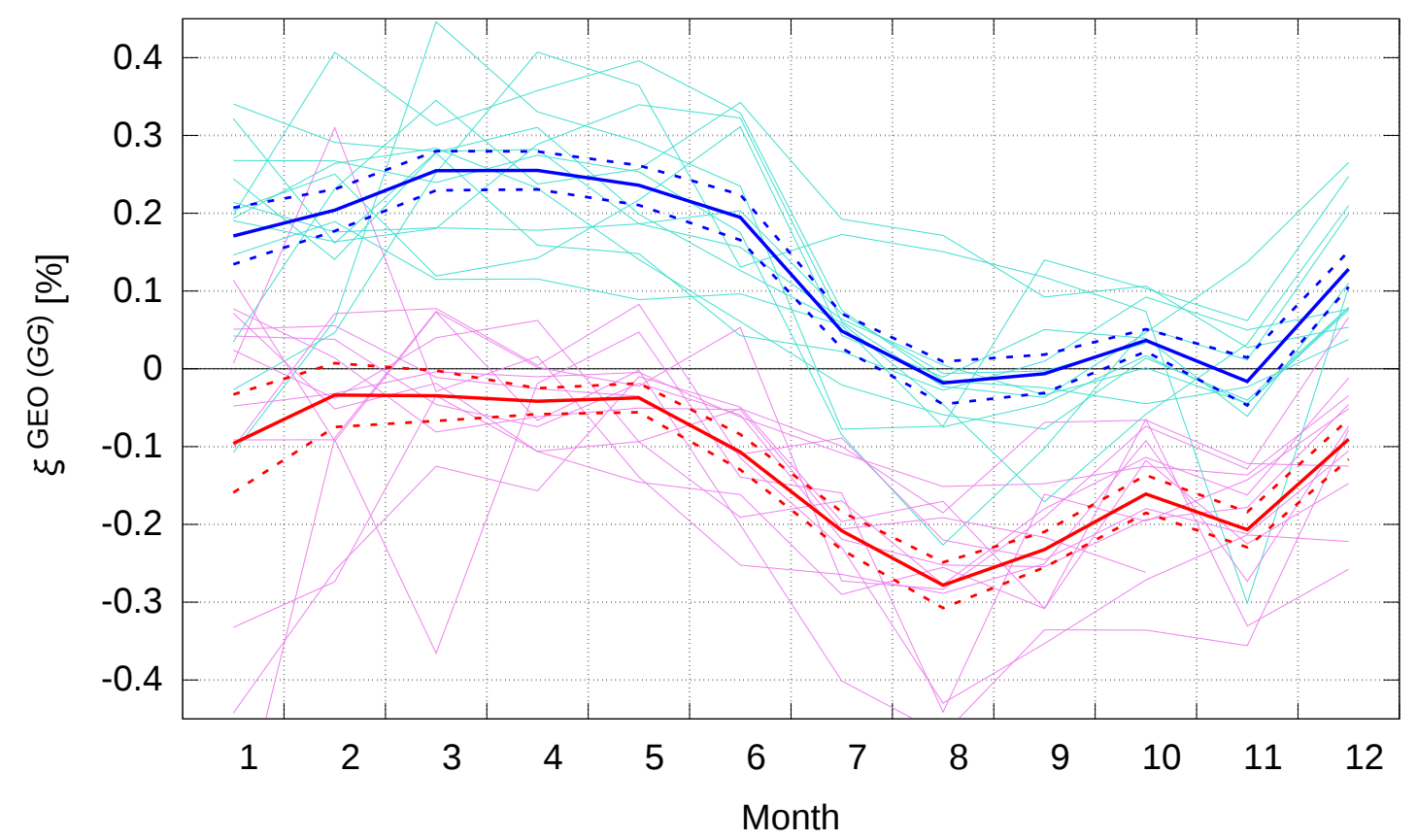

Figure 1: Average annual variations of monthly mean NSA $\left(\xi_{z}^{\mathrm{GEO}(G G)}\right)$ derived from the GG-component in toward (blue curves) and away (red curves) IMF sectors during 13 years between 2002 and 2014. Each light curve displays annual variation in each year, while each solid curve shows the average of all years. Two broken curves above and below the solid curve bound the standard error of the average evaluated from the dispersion of light curves in each IMF sector.

common zenith angle (49 degree) of the central viewing direction in the atmosphere, but the geomagnetic cut-off rigidities for these channels are different depending on the azimuth angle of each viewing direction. The GG-component in equation (2.2) is designed to minimize the temperature effect remaining in each of two differences due to the difference in the cut-off rigidities. From the GG-component, we can obtain the NSA in space, as

$$
\xi_{z}^{\mathrm{GEO}(G G)}=G G /\left(2 c_{1, \mathrm{~N} 2}^{0}-c_{1, \mathrm{~S} 2}^{0}-c_{1, \mathrm{E} 2}^{0}\right)=G G * 1.314
$$

where $c_{1, X X}^{0}$ is the coupling coefficient for the directional channel XX given by assuming the rigidity independent spectrum of the anisotropy. We calculate the daily mean $\xi_{z}^{\mathrm{GEO}(G G)}$ in $\mathrm{T}$ and $\mathrm{A}$ sectors from hourly data in the same manner as described in the section 2.1 for the GMDN data analysis. Okazaki et al. (2008) [2] showed that the day-to-day variation of $\xi_{z}^{\mathrm{GEO}(G G)}$ followed well the variation of $\xi_{z}^{\mathrm{GEO}}$ by the GMDN, while the hourly value of $\xi_{z}^{\mathrm{GEO}(G G)}$ showed larger fluctuation. This larger fluctuation of $\xi_{z}^{\mathrm{GEO}(G G)}$ is partly due to the statistical error of the GG-component in equation (2.2), but it is also due to the contribution from the equatorial anisotropy contained in the GG-component.

Figure 1 shows the average $\xi_{z}^{\mathrm{GEO}(G G)}$ in every month. A significant seasonal variation is seen with a maximum in March-April and a minimum in August-September in both $\mathrm{T}$ (blue curve) and A (red curve) sectors. We find that this seasonal variation is due to the contribution from the 


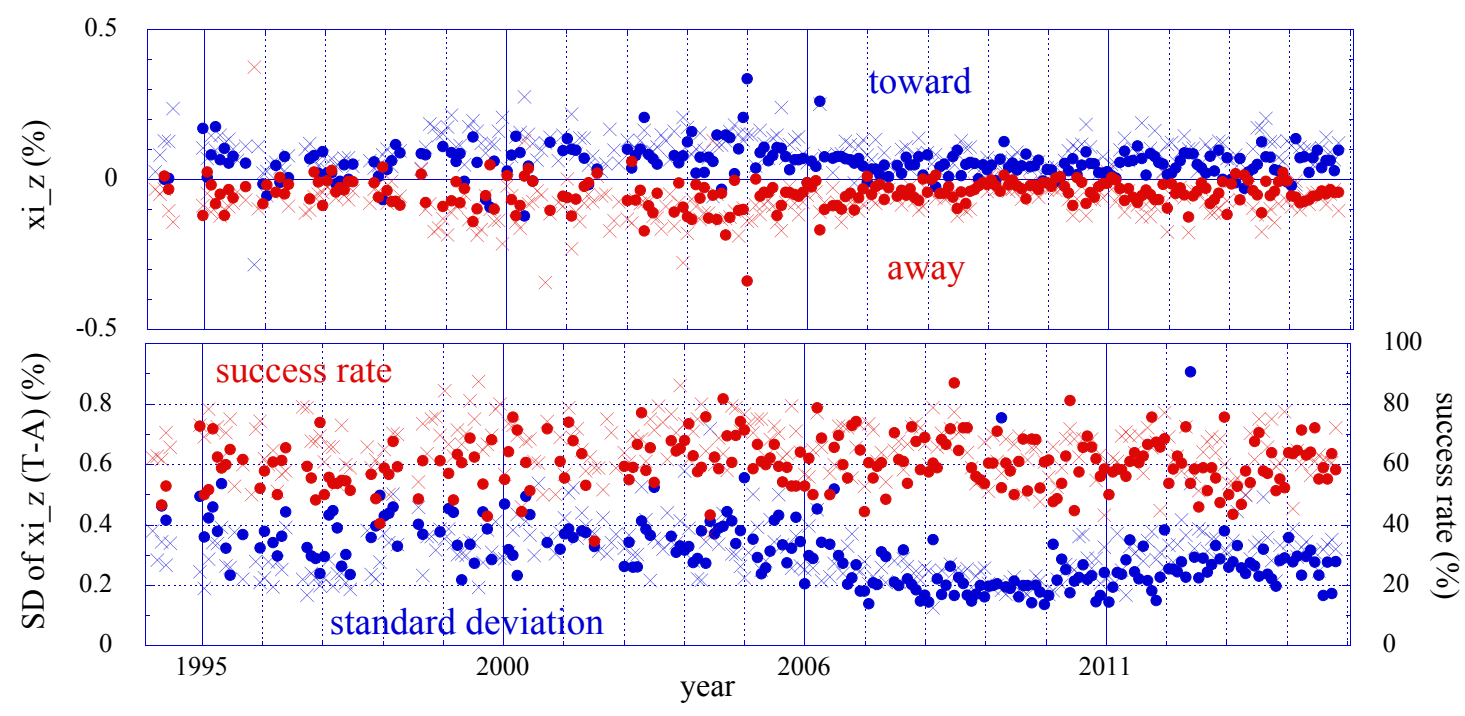

Figure 2: Temporal variations of Carrington Rotation average and standard deviation of NSA. Upper panel displays CR averages of $\xi_{z}^{\mathrm{GEO}}$ (solid circles) and $\xi_{z}^{\mathrm{GEO}(G G)}$ (crosses) each as a function of time (year) on the horizontal axis. Average NSAs in away and toward sectors in each CR are shown in red and blue characters, respectively. Blue solid circles and crosses in the lower panel display the standard deviation of daily mean NSA in each CR on the left vertical axis, while red characters display the "success rate" on the right vertical axis (see text). Solid circles and crosses in the lower panel also shows the data derived from $\xi_{z}^{\mathrm{GEO}}$ and $\xi_{z}^{\mathrm{GEO}(G G)}$ data, respectively.

anisotropy components in the ecliptic plane. Because of the 23.5 degree inclination of the Earth's rotation axis from the ecliptic plane, the average anisotropy in the ecliptic plane has maximum projections parallel to $\xi_{z}^{\mathrm{GEO}(G G)}$ in March-April and anti-parallel in August-September, respectively. It is confirmed quantitatively that the seasonal variation in Figure 1 is consistent with the average anisotropy in the ecliptic plane observed with the GMDN and transformed to the GSE coordinate system. This is a clear evidence of that the NSA in the GEO coordinate system is influenced by the anisotropy in the ecliptic plane.

\subsection{Long term variations of the NSA}

The upper panel of Figure 2 shows average $\xi_{z}^{\mathrm{GEO}}$ (sold circles) observed with the GMDN and average $\xi_{z}^{\mathrm{GEO}(G G)}$ (crosses) derived from the GG-component in away (red) and toward (blue) IMF sectors in every CR between CR1877 and CR2157 which spans 22-years between December 1993 and November 2014. In this figure, the average value in each rotation is also subtracted from $\xi_{z}^{\mathrm{GEO}(G G)}(t)$. A significant correlation is seen between the temporal variations of $\xi_{z}^{\mathrm{GEO}}$ and $\xi_{z}^{\mathrm{GEO}(G G)}$ indicating that overall variations observed with the GG-component and GMDN are consistent with each other. It is clear that $\xi_{z}^{\mathrm{GEO}(G G)}$ and $\xi_{z}^{\mathrm{GEO}(G G)}$ in toward (away) sector are positive (negative) in most rotations, as expected from the drift streaming expressed by a vector product $\left(\mathbf{B} \times \mathbf{G}_{r}\right)$ between the IMF vector $(\mathbf{B})$ and the radial density gradient $\left(\mathbf{G}_{r}\right)$ which points outward on average. It is also seen in both $\xi_{z}^{\mathrm{GEO}}$ and $\xi_{z}^{\mathrm{GEO}(G G)}$ that the separation between NSAs in toward and away sectors has minima around 1997 and 2009 near the solar activity minima. 


\section{Summary and discussions}

In the lower panel of Figure 2, blue solid circle and cross show the standard deviations (SDV) of daily mean $\xi_{z}^{\mathrm{GEO}}$ and $\xi_{z}^{\mathrm{GEO}(G G)}$ in each rotation, respectively. It is interesting to see that the standard deviation also has minima near the solar activity minima in accord with the toward-away separation of NSAs in sectors in the upper panel. This implies the ratio of the toward-away separation to the SDV is almost constant, without showing any clear minima (maxima) in the correlation with the solar activity. Laurenza et al. (2003) [11] analyzed daily mean GG-component recorded in 26 years from 1971 through 1997 and examined the "success rate" (SR) which is a ratio of the number of days when the sign of the observed $\xi_{z}^{\mathrm{GEO}}$ is positive (negative) in the toward (away) IMF sector to the total number of days and is introduced as a parameter indicating to what extent we can infer the IMF sector polarity from the sign of the observed NSA. If we assume normal distributions of $\xi_{z}^{\mathrm{GEO}}$ and $\xi_{z}^{\mathrm{GEO}(G G)}$ in each rotation, the SR is determined by the ratio of the toward-away separation to the SDV. Red solid circle (cross) in the lower panel of Figure 2 shows SR of daily mean $\xi_{z}^{\mathrm{GEO}}\left(\xi_{z}^{\mathrm{GEO}(G G)}\right)$ in each CR rotation on the right vertical axis. It is seen that there is no clear minimum and maximum seen with an overall SR (crosses) about $70 \%$ which is roughly consistent with the rate obtained by [11].

\section{Acknowledgments}

This work is supported in part by the joint research programs of the Solar-Terrestrial Environment Laboratory (STEL), Nagoya University and the Institute for Cosmic Ray Research (ICRR), University of Tokyo. The observations are supported by Nagoya University with the Nagoya multidirectional muon detector and CNPq, CAPES, INPE and UFSM with the Sao Martinho muon detector. The Kuwait muon telescope is supported by the project SP01/09 of the Research Administration of Kuwait University. The OMNI data were obtained from the GSFC/SPDF OMNIWeb interface at http://omniweb.gsfc.nasa.gov.

\section{References}

[1] Parker, E. N., 1965, Planet. Space Sci. 13, 9.

[2] Okazaki, Y. et al., 2008, Astrophys. J. 681, 693.

[3] Rockenbach, M. et al., 2014, Space Sci. Rev. 182, 1.

[4] Bieber, J. W., \& Chen, J. 1991, Astrophys. J. 372, 301.

[5] Chen, J., \& Bieber, J. W. 1993, Astrophys. J. 405, 375.

[6] Munakata, K. et al., 2014, Astrophys. J., 791, 22.

[7] Bieber, J. W., \& Pomerantz, M. A. 1986, Astrophys. J. 303, 843.

[8] Yasue, S. 1980, J. Geomag. Geoelectr., 32, 617.

[9] Swinson, D. B. 1969, J. Geophys. Res., 74, 5591.

[10] Mori, S., \& Nagashima, K., 1979, Planet. Space Sci., 27, 39.

[11] Laurenza, M. et al., 2003, J. Geophys. Res. 108, 1069. 
[12] Kozai, M. et al., 2014, Earth, Planets and Space 66, 151.

[13] Kuwabara, T. et al., 2009, J. Geophys. Res. 114, A05109.

[14] Fushishita, A. et al., 2010, Astrophys. J. 715, 1239.

[15] Murakami, K. et al., 1979, IL Nuovo Cimento 2C, 635.

[16] Nagashima, K., 1971, Rep. Ionos. Space Res. Japan 25, 189.

[17] Fujimoto, K., et al., 1984, Rep. Cosmic-Ray Res. Lab. 9.

[18] King, J. H., \& Papitashvili, N. E., 2005, J. Geophys. Res. 110, A02104. 